\section{Commentary: How to create a cardiac surgery simulator in the era of two-day shipping}

\author{
Peter D. Drevets, MD, ${ }^{a}$ and \\ Richard Lee, MD, JD, MBA ${ }^{\mathrm{b}}$
}

Simulation is a prevalent topic within modern surgical training. Kelly and colleagues ${ }^{1}$ present the results of a survey sent to cardiothoracic trainees regarding their experience and feelings towards simulation. Using the Thoracic Surgery Resident's Association e-mail list, the authors distributed a survey to current cardiothoracic trainees, with a response rate of $24.7 \% .^{1}$ The majority of respondents believed that practice outside the operating room was important $(98 \%)$ and improved their operative skills $(93 \%){ }^{1}$ The biggest obstacle to simulation practice was lack of time $(78 \%)$, with most trainees reporting practicing 2 or fewer hours per week $(71 \%){ }^{1}$ Other factors that inhibited nonoperative simulation were lack of instruction $(47 \%)$ and lack of materials $(40 \%){ }^{1} \quad$ To address these barriers to simulation, the authors describe prototype do-ityourself simulation devices that can be made using easily obtained materials via the internet or the local hardware store. A list of starting materials available entirely on Amazon is provided. With the materials, the trainee may create 2 different devices that simulate the basic elements of cardiac surgery, such as cannulation, suture sills, and anastomosis. Once created, the specific device becomes a portable trainer that can be used in-between cases, at home, and wherever else the trainee should want to practice.

There are several looming questions. First is the accuracy with which the devices simulate their intended real-world skill. This will require study validation by both trainees and established surgeons. Second is whether the proposed

From the ${ }^{\mathrm{a}}$ Department of Surgery, and ${ }^{\mathrm{b}}$ Division of Cardiothoracic Surgery, Medical College of Georgia, Augusta University, Augusta, Ga

Disclosures: The authors reported no conflicts of interest.

The Journal policy requires editors and reviewers to disclose conflicts of interest and to decline handling or reviewing manuscripts for which they may have a conflict of interest. The editors and reviewers of this article have no conflicts of interest.

Received for publication May 26, 2021; revisions received May 26, 2021; accepted for publication June 1, 2021; available ahead of print June 11, 2021.

Address for reprints: Richard Lee, MD, JD, MBA, Division of Cardiothoracic Surgery, Augusta University, 1120 15th St, BA-4300, Augusta, GA 30912 (E-mail: ricklee@augusta.edu).

JTCVS Techniques 2021;8:112-3

2666-2507

Copyright (C) 2021 The Author(s). Published by Elsevier Inc. on behalf of The American Association for Thoracic Surgery. This is an open access article under the CC BY-NC-ND license (http://creativecommons.org/licenses/by-nc-nd/4.0/).

https://doi.org/10.1016/j.xjtc.2021.06.005

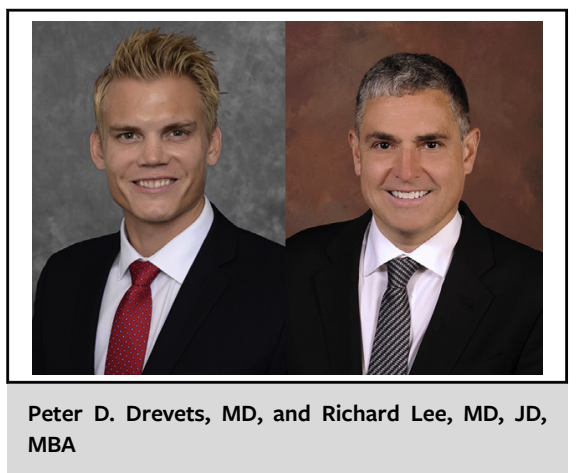

CENTRAL MESSAGE

Cardiothoracic trainees believe that practice outside the operating room is important. Portable training devices created from household items increase access to cardiac surgery simulation.

devices fill an existing need. A direct comparison of the authors' model to similar simulation devices is needed. Existing simulators, like the Ramphal Cardiac Surgery Simulator, rely on porcine material and require extensive setup. Certainly, no trainee has access to a Ramphal simulator in their home, and the potential portability of the authors device is appealing. However, with cadaver laboratories, simulation laboratories, and education sessions available, is there a niche to fill? Finally, will timestressed trainees actually be interested in using the device? More material for additional study.

The authors suggest that in the coronavirus disease 2019 era, virtual platforms are important to surgical training. While we are currently in the coronavirus disease 2019 era now, we likely will not be in the future. Virtual and home training sessions may struggle to maintain relevance after the end of the current pandemic. The proposed use of the device in-between cases to practice a recently learned skill is interesting, provided the device is effective and superior to existing equipment available in the hospital setting. More intriguing are the authors' comments on the possibility of remote teaching via video-based applications. This possibility is not unique to this device, however.

Without study validation, the proposed devices will likely remain an interesting idea. However, many great ideas have humble beginnings. Prospective studies are forthcoming, and the results will be interesting. The creativity and innovative thinking exhibited by the authors is excellent and should 
be encouraged. Amazon began in a garage, there is no reason the next device to revolutionize cardiac surgical simulation cannot arise from a similar origin.

\section{Reference}

1. Kelly JJ, Han JJ, Patrick WL, Mays JC, Iyengar A, Helmers MR, et al Do-it-yourself simulators and building a culture of practice in the virtual era. J Thorac Cardiovasc Surg Tech. 2021;8:100-11. 